\title{
Social determinants of health associated with poor outcome for rural patients following resected pancreatic cancer
}

\author{
Quyen D. Chu ${ }^{1}$, Mei-Chin Hsieh ${ }^{2}$, John F. Gibbs ${ }^{3} \wedge$, Xiao-Cheng Wu ${ }^{2}$ \\ ${ }^{1}$ Departments of Surgery, LSU Health Sciences Center-Shreveport, Shreveport, Louisiana, USA; ${ }^{2}$ Louisiana Tumor Registry \& Epidemiology and \\ School of Public Health at LSU Health Sciences-New Orleans, New Orleans, Louisiana, USA; ${ }^{3}$ Hackensack Meridian School of Medicine, Nutley, \\ NJ, USA \\ Contributions: (I) Conception and design: All authors; (II) Administrative support: All authors; (III) Provision of study materials or patients: All \\ authors; (IV) Collection and assembly of data: MC Hsieh, XC Wu; (V) Data analysis and interpretation: All authors; (VI) Manuscript writing: All \\ authors; (VII) Final approval of manuscript: All authors. \\ Correspondence to: John F. Gibbs, MD, MHCM. Medical Director of Oncology for Southern Region, Hope Tower Oncology Administration, 19 Davis \\ Ave, Neptune, NJ, 07731, USA. Email: John.Gibbs@HMHN.org.
}

Background: The impact of rurality on outcome for patients who had resected pancreatic ductal adenocarcinoma (PDAC) is unclear. We hypothesize that poor outcomes for rural patients are associated with adverse social determinants of health $(\mathrm{SDoH})$. The objective of this study is to assess the difference in overall survival (OS) of PDAC patients between rural, urban, and contributing factors.

Methods: A cohort of 25,536 patients diagnosed with stage I-III pancreatic adenocarcinoma from 2003 to 2011 and underwent resection were evaluated from the National Cancer Database. Socioeconomic/ demographic, clinicopathological, and treatment variables were compared between rural and urban residences. The 5-year OS was calculated using the Kaplan-Meier method. The Cox regression model was used to assess factors associated with OS. P value $<0.05$ was considered significant.

Results: In univariate analysis, the rural residence was a predictor of poor OS. The 5-year OS for rural $(\mathrm{N}=4,389)$ and urban $(\mathrm{N}=21,147)$ was $18.8 \%$ (95\% CI: $17.4-20.2 \%)$ and $22.3 \%$ (95\% CI: $21.6-22.9 \%$; $\mathrm{P}<0.0001)$, respectively. The risk of all causes of death was $10.3 \%$ higher $(\mathrm{P}<0.0001)$ in rural than urban patients. In multivariable analysis, rurality was not an independent predictor of $\mathrm{OS}(\mathrm{P}=0.407)$. Independent predictors of worse OS included adverse social determinants of health associated with the rural population and these included a low income $(\mathrm{P}<0.0001)$, low education level $(\mathrm{P}<0.01)$, low insurance status $(\mathrm{P}<0.01)$, and treatment at a low-volume facility $(\mathrm{P}<0.0001)$.

Conclusions: Rural/urban outcome disparities for resected stage I-III pancreatic cancer outcome can be explained by adverse social determinants of health associated with rural population.

Keywords: Pancreatic cancer; outcome; rural; urban; disparities

Submitted Dec 10, 2020. Accepted for publication Jun 08, 2021.

doi: 10.21037/jgo-20-583

View this article at: https://dx.doi.org/10.21037/jgo-20-583

\section{Introduction}

Pancreatic ductal adenocarcinoma (PDAC) is a formidable disease, and its outcome is one of the worst amongst all of the malignancies. Although it comprises only $3 \%$ of cancer diagnoses, it contributes to $7 \%$ of cancer deaths (1). PDAC is the fourth leading cause of cancer-related deaths in the United States (U.S.) and unfortunately, is expected to become the second-leading cause of cancer death by 2030

\footnotetext{
$\wedge$ ORCID: 0000-0003-0037-8014.
} 
$(2,3)$. It is estimated that in $2020,57,600$ people in the U.S. will be diagnosed with this malignancy, of which 47,050 people will die of it (4). Surgery remains the only treatment for potential cure.

Outcomes following a pancreatectomy depend on a myriad of factors. If found at an early-stage patient, clinical, treatment, hospital, and socioeconomic factors all play a profound role in cancer outcomes. While there is ample data on how a number of these factors have affected cancer outcome, there is a dearth of data on the impact of rurality on outcomes, especially for those with resected pancreatic cancer. What is not clear is whether outcomes for rural patients who underwent a successful pancreatectomy are different from those from urban region, and if so, what factors are contributing to such a disparity?

Up to $19 \%$ of the U.S. population or 59 million people reside in rural communities (5). Rural Americans suffer many inequities compared to urban Americans. People of rural communities face a myriad of socioeconomic and demographic challenges that negatively affect their health. Compared to urban communities, rural communities have higher poverty rates, higher proportion of the elderly population, and lower educational level. They also lack access to adequate health services, and their community lacks the resources to maintain adequate public health infrastructures (6).

A recent CDC report revealed that rural patients have worse cancer outcomes than their urban counterparts (5). Although cancer mortality rates have declined nationwide, such a decline has not been uniformed for all subgroup of patients. For rural communities, the rate of decline was actually slower than that for urban communities $(1.0 \% \mathrm{vs}$. $1.6 \%$ per year). Despite having lower cancer incidence rates (442 cases per 100,000 persons) than their urban counterparts (457 cases per 100,000 persons), rural death rates were higher (180 deaths per 100,000 persons vs. 158 deaths per 100,000 persons) (5). Death per incidence rate for rural is $40.7 \%$ vs. $34.6 \%$ for urban.

Emerging data strongly support a link between cancer outcomes and social determinants of health $(\mathrm{SDoH})(7,8)$. The World Health Organization (WHO) defines SDoH as "the conditions in which people are born, grow, live, work and age" and that "these circumstances are shaped by the distribution of money, power, and resources at global, national and local levels" (9). Health inequities among different groups of individuals are due to differences in SDoH. While optimal treatment such as surgery and chemo and/or radiation therapy may provide the patient a chance for long-term survival, such medical care accounts only $10 \%$ to $20 \%$ of the variation in years of life lost $(10,11)$; the remaining $80-90 \%$ is dependent on $\mathrm{SDoH}(12,13)$. In essence, adverse $\mathrm{SDoH}$ that are associated with a particular group of individuals may partly account for why one patient has a poorer long-term survival than another patient, despite both having received optimal surgical care.

The association between pancreatic cancer outcomes and rurality as a driver of health inequity has not been fully investigated. Given such limitations, we sought to (I) understand the dynamics between urban-rural residency and compare survival of resected PDAC patients between rural and urban patients, (II) identify factors resulted from the difference. To our knowledge, this is the first nationwide U.S. hospital-based study to perform such an analysis. We believe that rural patients with resected pancreatic cancer possess unique characteristics that pose a challenge for them to reach parity in outcome with their urban counterparts. We also believe that there are adverse $\mathrm{SDoH}$ associated with rural residence and by recognizing these drivers; we would be able to better inform policymakers and stakeholders to address the rural/urban disparities.

We present the study in accordance with the STROBE reporting checklist (available at https://dx.doi.org/10.21037/ jgo-20-583).

\section{Methods}

The study was conducted in accordance with the Declaration of Helsinki (as revised in 2013). The study was exempted from Institutional Review Board (IRB) approval by the Louisiana State University Health Sciences CenterShreveport.

\section{Data source}

The National Cancer Database (NCDB) is a joint project of the Commission on Cancer $(\mathrm{CoC})$ of the American College of Surgeons and the American Cancer Society. Approximately $70 \%$ of newly diagnosed cancer cases in the U.S and 30 million historical records are captured in the database. The data sets in the participant user file (PUF) were de-identified and were in compliant with the privacy requirements of the Health Insurance Portability and Accountability Act (HIPAA).

\section{Study population}

A cohort of 25,536 patients who were diagnosed with 
resected stage I-III pancreatic adenocarcinoma (ICD-03; C25.0-C25.9) between 2003 and 2011 were analyzed to determine significant factors associated with overall survival (OS) (14). According to the NCDB's PUF dictionary, the treating pancreatic surgical facility type was classified as (I) community cancer program (CCP), (II) comprehensive community cancer program (CCCP), (III) academic research program (ARP), and (IV) others (15). Community cancer programs are those that treat between 100 and 500 newly diagnosed cancer patients each year, and patients may be referred to another facility for part of their diagnosis or treatment (15). Comprehensive community cancer programs are those that treat more than 500 newly diagnosed cancer patients each year (15). Academic and research institutions are those that treat more than 500 newly diagnosed cancer patients each year while offering postgraduate medical education programs (15).

Race was limited to White and Black. Insurance status was classified as (I) uninsured, (II) private insurance/ managed care, (III) Medicaid, (IV) Medicare, and (V) other governmental insurance. Patients with both private insurance and Medicare were grouped in the private insurance category. Median household income level was classified as (I) $<\$ 38,000$, (II) $\$ 38,000-\$ 47,999$, (III) $\$ 48,000-\$ 62,999$, and (IV) $\geq \$ 63,000$. Education level was classified into percentage of adults (age $\geq 25$ years) who did not graduate from high school in the area based on the 2012 American Community Survey data: (I) $\geq 21 \%$, (II) 13-20.9\%, (III) 7-12.9\%, and (IV) $<7 \%$. Charlson/Deyo comorbidity score has been recorded since 2003 and was reported as zero, one or two $(16,17)$.

We used the urban-rural 2013 continuum classification (United State Department of Agriculture Economic Research Service, http://www.ers.usda.gov/data-products/ rural-urban-continuum-codes) which distinguishes metropolitan counties by the population size of their metro area, and nonmetropolitan counties by degree of urbanization and adjacency to a metro area to define urban and rural area. The urban area included counties that were categorized as metropolitan and rural area contained counties other than metropolitan counties.

Great circle distance is measured along the surface of the sphere and defined as the shortest distance in miles $(<50 v s$. $\geq 50$ miles) between the patient's residence at diagnosis and the hospital that reported the case.

Annual hospital volume (AHV) was defined as the average number of procedures per year reported to the NCDB. The mean AHV of pancreatectomies was calculated for currently accredited $\mathrm{CoC}$ hospitals during the study period (1998 to 2011), and grouped as four categories: $<5$ patients, 5-9 patients, $10-19$ patients and $\geq 20$ patients.

\section{Statistical analysis}

Descriptive statistics for the different variable were reported. Univariate analysis of each categorical variable was performed using chi-square test. The Kaplan-Meier method was used for survival analysis. Cox proportional hazards regression was used to identify factors significantly associated with the risk of deaths for all causes and the hazard ratios (HR) and confidence intervals (CI) were calculated. A P value $<0.05$ was considered statistically significant. All statistical analyses were performed using SAS Version 9.4 statistical software, (SAS Institute Inc., Cary, NC, USA, 2013).

\section{Results}

\section{Demographics and clinical characteristics of patients who underwent pancreatectomy}

The median follow-up was 18.8 months. Summary statistics on patient characteristics and treatment outcomes are shown in Table 1. The median age of the entire cohort was 66 years (range, 18-90 years). There are 1,928 (7.6\%) patients lost to follow-up. There were differences in baseline clinical characteristics between rural and urban patients. There was a preponderance of Caucasian living in rural regions compared to African-American. However, compared to urban communities, rural communities were more likely $(\mathrm{P}<0.01)$ to belong in the lower income bracket (76.2\% had income $<\$ 48,000$ in rural vs. $33.9 \%$ for urban), to attain low education level, to be uninsured, to be treated at community cancer program and low volume centers, to travel $\geq 50$ miles for their treatment, and more likely to have a higher comorbidity score. There were no significant differences in distribution of stage of disease or receipt of radiation between urban and rural regions.

\section{Unadjusted factors associated with overall survival}

Figure 1 shows the Kaplan-Meier OS curve for rural and urban patients who had pancreatectomy for stage I-III pancreatic cancer. The 5-year OS rate was $22.3 \%$ (95\% CI: $21.6-22.9 \%$ ) for urban region and $18.8 \%$ (95\% CI: $17.4-$ 
Table 1 Demographic and clinical characteristics of stage I-III resected pancreatic cancer patients by urban/rural status

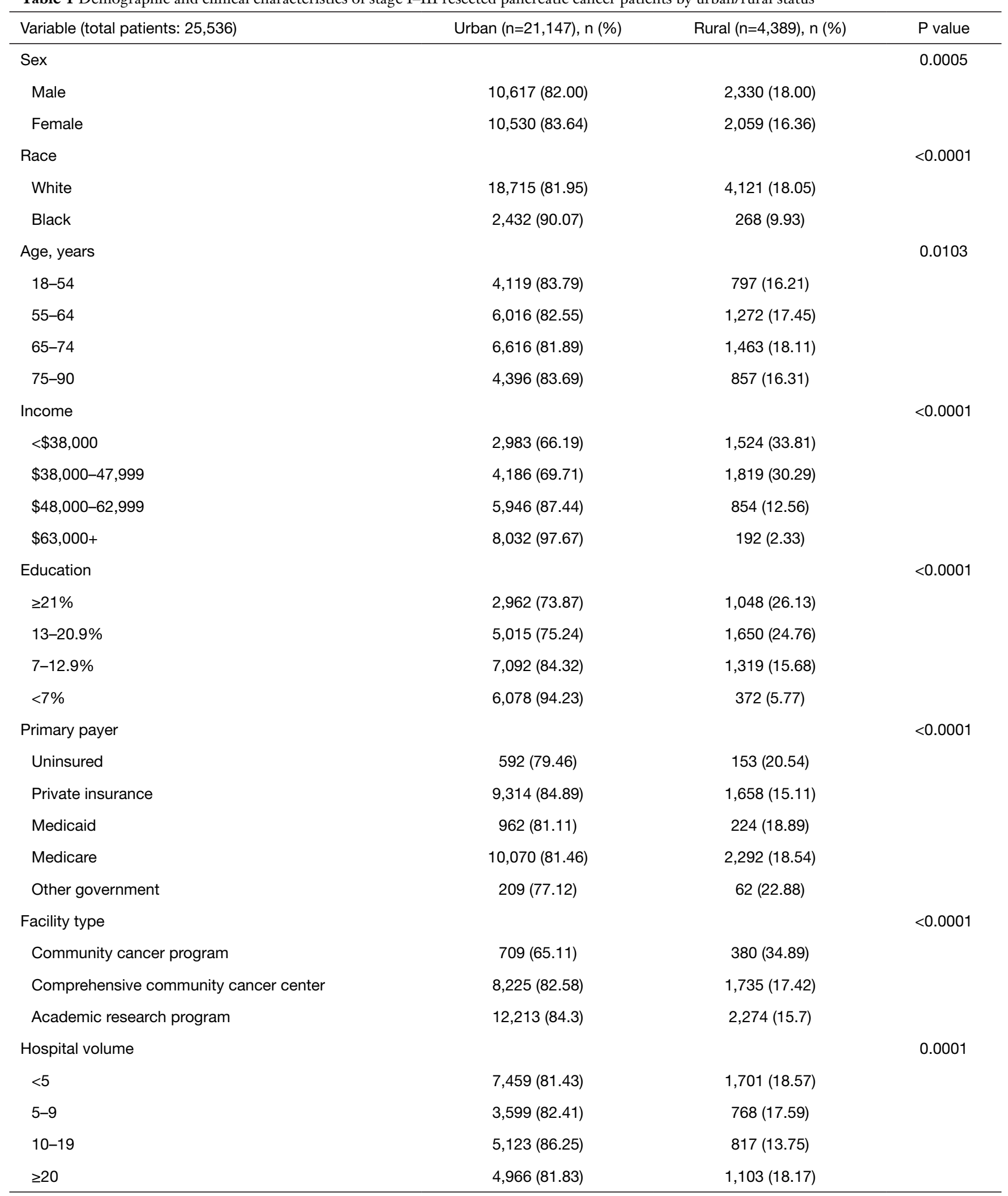

Table 1 (continued) 
Table 1 (continued)

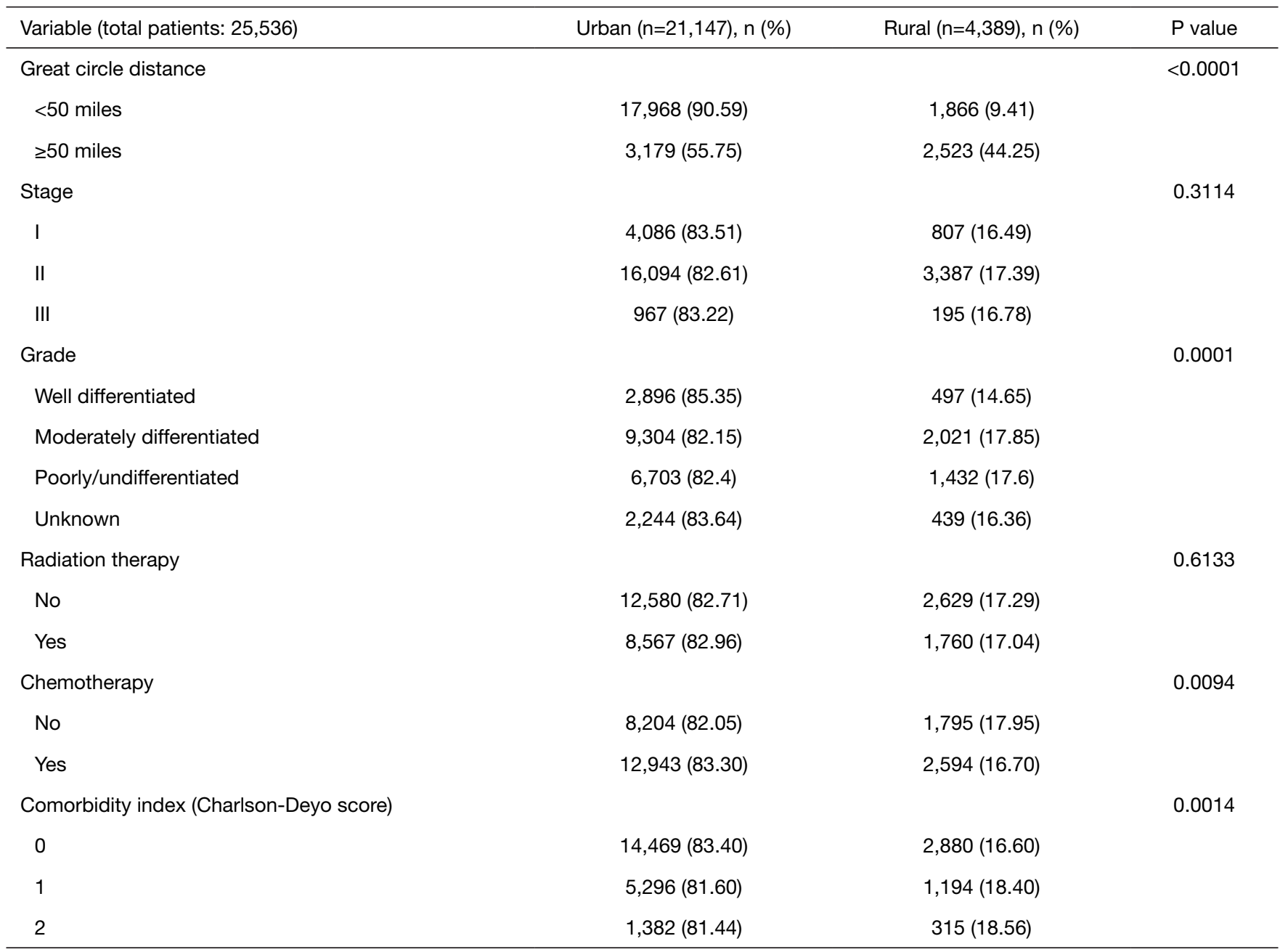

$20.2 \%)$ for rural region $(\mathrm{P}<0.0001)$. The median survival was 21.3 months (95\% CI: 20.9-21.7 months) for urban region and 19.1 months (95\% CI: 18.4-20.0 months) for rural region.

The risk of all causes of death was $10.3 \%$ higher for rural patients than urban patients (HR 1.103; 95\% CI: 1.061-1.146; $\mathrm{P}<0.0001)$. Those with an annual income $<\$ 48,000$ had a $16 \%$ to $23 \%$ higher risk of dying compared to those with an annual income $\geq \$ 63,000(\mathrm{P}<0.0001)$; those with limited education level had a $9 \%$ to $21 \%$ risk of dying compared to those with the highest level of education $(\mathrm{P}<0.0001)$; uninsured patients are at a $13 \%$ risk of dying compared to privately insured patients $(\mathrm{P}=0.013)$, Medicaid recipients are at a $17 \%$ risk of dying compared to privately insured patients $(\mathrm{P}<0.0001)$ and Medicare recipients are at a $40 \%$ risk of dying compared to privately insured patients
$(\mathrm{P}<0.0001)$; compared to patients at academic research program, patients at a community cancer program and comprehensive community cancer program are at a $17 \%$ and $14 \%$ risk of dying, respectively $(\mathrm{P}<0.0001)$; compared to patients treated at a hospital with volume $\geq 20$ patients per year, patients treated at a hospital with volume $<5$ per year and volume 5-9 per year were at $23 \%$ and $16 \%$ risk of dying, respectively $(\mathrm{P}<0.0001)$.

\section{Adjusted factors associated with overall survival}

After adjusting for patient's demographics (i.e., gender, race, age), access to care factors (i.e., insurance, hospital type, hospital volume, great circle distance), clinical factors (i.e., grade, stage, comorbidity, treatment), and $\mathrm{SDoH}$ (i.e., income, education), the difference in the risk of overall deaths 


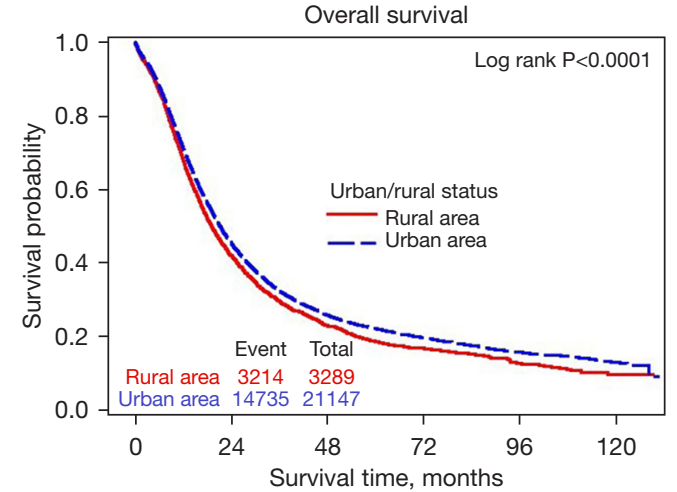

Figure 1 Kaplan-Meier OS curve for rural and urban patients who underwent pancreatectomy for stage I-III pancreatic cancer. The 5-year OS for urban and rural was 22.3\% (95\% CI: 21.6-22.9\%) and $18.8 \%$ (95\% CI: $17.4-20.2 \%$; $<0.0001$ ), respectively. The median survival time was 21.3 months (95\% CI: 20.9-21.7 months) for urban and 19.1 months (95\% CI: 18.4-20.0 months) for rural.

between rural and urban was no longer statistically significant and the following were independent predictors of poor OS: low-income level, low education attainment level, male gender, older age group, Medicaid/Medicare, low-volume facilities, higher stage, poor histology, high comorbidity index, and lack of chemotherapy receipt (Table 2).

Those with an annual income $<\$ 63,000$ had a $5 \%$ to $14 \%$ risk of dying compared to those with an annual income $\geq \$ 63,000$. Patients with low education level have a $6 \%$ to $11 \%$ increased risk of death compared to those with high education level; compared to patients with private insurance, Medicaid and Medicare recipients have a $11 \%$ and $15 \%$ risk of dying, respectively $(\mathrm{P}<0.001)$; patients treated at a hospital volume $<20$ cases per year are at a $6 \%$ to $26 \%$ risk of dying compared to those with $\geq 20$ cases per year.

To understand the role of the different factors, have on the rural/urban disparities for resected stage I-III pancreatic cancer, we generated five models (Table 3). In the first model, we adjusted for patients' demographics (i.e., gender, race, and age) and found that patients residing in rural area had a $10 \%$ risk of death (died of any cause) than those resided in urban areas (HR 1.099; 95\% CI: 1.058-1.142; $\mathrm{P}<0.0001)$. However, when this model was adjusted to include $\mathrm{SDoH}$ (i.e., income and education; Model 2), the rural-urban disparities no longer exist $(\mathrm{P}=0.2907)$. When adjusting for access to care (Model 3) or clinical factors (Model 4) to patient demographics, we see persistence of the rural-urban mortality disparities $(\mathrm{P}<0.01)$. However, when adjusted to include all factors, including SDoH, the rural-urban mortality disparities cease to exist (Model 5; $\mathrm{P}=0.407)$. This demonstrates that $\mathrm{SDoH}$ factors play an important role in the urban-rural mortality disparities among stage I-III pancreatic cancer patients.

\section{Discussion}

Everything being equal, outcome for a patient who had a pancreatectomy should be dependent on the biology of his/ her disease (i.e., stage of disease), success of surgery, and proper receipt of neoadjuvant/adjuvant therapy. However, other determinants such as income, education, also known as $\mathrm{SDoH}$, also have an impact on outcome. In fact, $\mathrm{SDoH}$ appears to have a greater influence on outcome than the medical care that the patient had received (10).

In our study of a large cohort of patients who underwent a pancreatectomy for pancreatic cancer, we found that $\mathrm{SDoH}$ which had an adverse effect on outcome were low income, low education level, low insurance status, and treatment at a low-volume facility; all of these adverse SDoH were associated with rural population.

$\mathrm{SDoH}$ are the conditions which shape the health of individuals (9). The importance of addressing $\mathrm{SDoH}$ is underscored by the fact that despite the U.S. spending significantly more per capita and in total on health care compared to other high-income countries; it has lowerthan-average outcomes across a wide range of health indicators (18). Notwithstanding, the U.S. has the lowest life expectancy rate and highest infant mortality rate (18). In addition, because of suboptimal prevention efforts, many patients developed chronic health conditions and various complications, which also contribute to poor outcomes. Therefore, high health care spending does not necessarily indicate better quality health care.

Interestingly enough, the total social spending in the U.S. is below the average of other high-income countries (16.7\% vs. 19.4\%), meaning that although patients may have access to quality health care, they may not have easy access to food, transportation, or safe housing, situations that lead to poor health outcomes $(18,19)$. Furthermore, rural patients may not be able to access the same quality of health care as urban patients. Rural hospitals and clinics lack multidisciplinary experts and good medical equipment. Table 1 shows that urban patients are more likely to be treated at Academic Research Programs and high-volume hospitals than rural patients do.

In an editorial, Steven Woolf commented that most of 
Table 2 Factors associated with overall survival in stage I-III resected pancreatic cancer patients

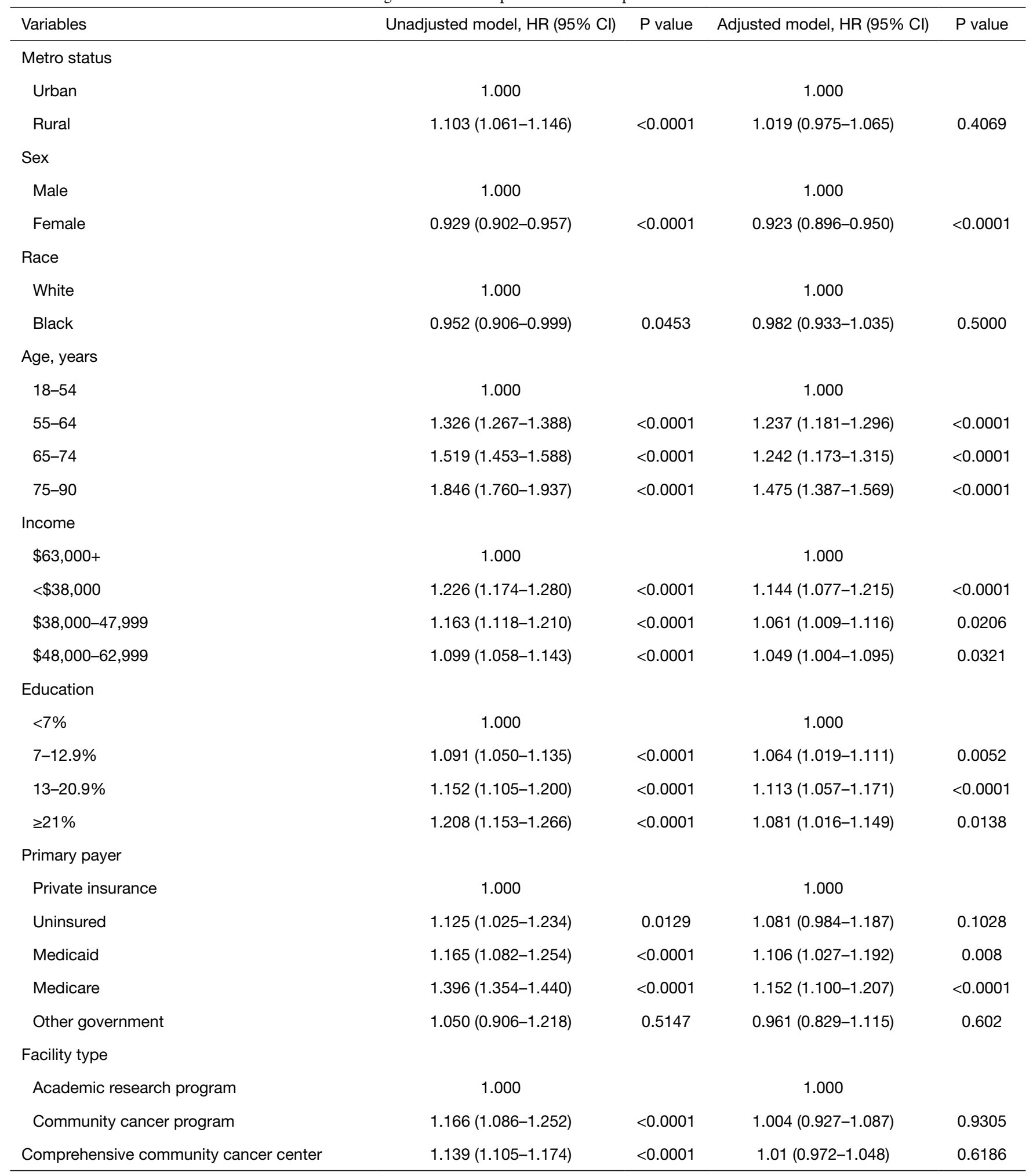

Table 2 (continued) 
Table 2 (continued)

\begin{tabular}{|c|c|c|c|c|}
\hline Variables & Unadjusted model, HR (95\% Cl) & $P$ value & Adjusted model, HR (95\% Cl) & $P$ value \\
\hline \multicolumn{5}{|l|}{ Hospital volume } \\
\hline$\geq 20$ & 1.000 & & 1.000 & \\
\hline $10-19$ & $1.031(0.987-1.077)$ & 0.1695 & $1.057(1.011-1.104)$ & 0.0147 \\
\hline $5-9$ & $1.161(1.108-1.217)$ & $<0.0001$ & $1.159(1.102-1.219)$ & $<0.0001$ \\
\hline \multicolumn{5}{|l|}{ Great circle distance } \\
\hline$<50$ miles & 1.000 & & 1.000 & \\
\hline$\geq 50$ miles & $0.988(0.954-1.024)$ & 0.5209 & $0.966(0.926-1.007)$ & 0.1041 \\
\hline \multicolumn{5}{|l|}{ Stage } \\
\hline III & $3.329(3.090-3.586)$ & $<0.0001$ & $3.200(2.968-3.451)$ & $<0.0001$ \\
\hline \multicolumn{5}{|l|}{ Grade } \\
\hline Well differentiated & 1.000 & & 1.000 & \\
\hline Moderately differentiated & $2.212(2.093-2.337)$ & $<0.0001$ & $2.019(1.909-2.136)$ & $<0.0001$ \\
\hline Poorly/undifferentiated & $2.900(2.740-3.068)$ & $<0.0001$ & $2.650(2.501-2.808)$ & $<0.0001$ \\
\hline Unknown & $1.280(1.191-1.375)$ & $<0.0001$ & $1.335(1.242-1.435)$ & $<0.0001$ \\
\hline \multicolumn{5}{|l|}{ Radiation therapy } \\
\hline Yes & 1.000 & & 1.000 & \\
\hline 0 & 1.000 & & 1.000 & \\
\hline 1 & $1.151(1.112-1.190)$ & $<0.0001$ & $1.105(1.068-1.143)$ & $<0.0001$ \\
\hline 2 & $1.390(1.312-1.472)$ & $<0.0001$ & $1.289(1.217-1.366)$ & $<0.0001$ \\
\hline
\end{tabular}

the influences on an individual's health lie outside of the clinic. Medical/surgical care accounts for only $5 \%$ to $20 \%$ of variation in premature death, while the remaining 80-95\% are dependent on $\mathrm{SDoH}$ (10). This means that despite our ability to select patients for surgery, as well as our technical abilities and clinical expertise, 80-95\% of premature death are dependent on factors outside of our operating room and hospital. Thus, to have a major impact on improving the nation's health, we will need to recognize and address SDoH and not rely entirely on having more bench science, more drugs, or more spending on health care (10).

Among the different disparities that are associated with $\mathrm{SDoH}$ such as race/ethnicity, age, socioeconomic status, the rurality disparity is one of the least studied. However, emerging data found that rural communities across America possess a unique set of socioeconomic/demographic challenges that predispose them to having a worse health outcome. In general, these rural communities tend to have 
Table 3 Model demonstrating impact of social determinants of health on rural regions

\begin{tabular}{lcc}
\hline Adjusted model & HR $(95 \%$ Cl) & P value \\
\hline Model 1 & $1.099(1.058-1.142)$ & $<0.0001$ \\
Model 2 & $1.022(0.981-1.065)$ & 0.2907 \\
Model 3 & $1.069(1.024-1.115)$ & 0.0022 \\
Model 4 & $1.084(1.043-1.126)$ & $<0.0001$ \\
Model 5 & $1.019(0.975-1.065)$ & 0.4069 \\
\hline
\end{tabular}

Note that when adverse SDoH were excluded (Model 1, 3, 4); rurality becomes a significant predictor of outcome. However, when SDoH were added into the model (Model 2 and 5), rurality no longer plays a role in predicting outcome for patients with stage I-IIII pancreatic cancer. This suggests that SDoH was a confounder for the association between rurality and poor outcome. HR compared rural to urban (urban as reference). Model 1: adjusted for patient's demographics (sex, race, age); Model 2: adjusted for patient's demographics and SDoH (income and education); Model 3: adjusted for patient's demographics and access to care factors (insurance, hospital type, hospital volume, great circle distance); Model 4: adjusted for patient's demographics and clinical factors (stage, grade, treatment, and comorbidity); Model 5: adjusted for all factors.

higher poverty rates, lower educational attainment, and lack of access to health services. They also tend to lack adequate insurance coverage, have limited transportation, lack access to preventive and screening services, and have attributes or are engaged in behaviors that are associated with cancer such as tobacco use, alcohol consumption, obesity, less physical activity, lower HPV vaccination rates, and less-frequent adoption of sun safety measures. Consequently, not only do they have higher average death rates than those living in urban communities, they also have higher incidence and death rates for cancers associated with smoking (i.e., lung and laryngeal) and those that can be prevented by screening (i.e., colorectal and cervical cancers).

Although multiple studies on health disparities in pancreatic cancer exist across a spectrum of socioeconomic typologies such as race/ethnicity, socioeconomic status, marital status, and insurance, very few have looked at the impact of rurality on pancreatic cancer outcome $(20,21)$. In fact, to our knowledge, only two studies reported the urbanrural disparities for pancreatic cancer, both of which were based on populations outside of the U.S. and even then, their results were very different from each other. Canale et al. evaluated a population of 659 patients from British Columbia with advanced pancreatic cancer and found that the risk of death was similar between urban and rural groups, even after adjusting for other covariates (20). However, Kirkegård et al. conducted a cohort study of 10,594 patients with pancreatic cancer from Denmark and reported that urban patients had better survivals than rural patients did, even after adjusting for potential confounders (21). The studied populations were also different. Canale's population were mainly those with metastatic disease, while Kirkegård's included patients with all stages of disease. In comparison, our study has a sizeable number of patients and we limited our study only to those who had undergone a resection. To our knowledge, our study is the first nationwide, U.S. hospital-based cohort. We restricted our study to this population to mitigate the impact of lack of access for surgical resection on outcome.

Unlike previous studies that report disparities along racial/ethnic fault lines, our study found that the rural/urban outcome disparities for resected pancreatic cancer affected mainly Caucasian patients as over $93 \%(4,121 / 4,389)$ of pancreatic cancer patients in rural areas were Caucasians $(12,22,23)$. This may reflect the nature of $\mathrm{NCDB}$, since data show that $90 \%$ of the cases are Caucasians; Caucasian patients are more likely than African-American patients to receive care at $\mathrm{CoC}$ hospitals since racial and ethnic minorities, in general, make up $19 \%$ of non-metro residents. Additionally, most of the rural patients belonged to the socioeconomically disadvantaged strata. This study, therefore, sheds lights on the subject of disparity, mainly that rural population has a unique set of challenges that should be recognized and understood if we are to reach outcome parity for everyone affected by this deadly cancer.

We found that rural patients possess particular set of social determinants that drive adverse health outcomes. Compared to urban patients, rural patients who underwent a pancreatectomy were more impoverished, had lower education level, were less likely to have private insurance, and were more likely to be uninsured. It is well established that high-volume centers have better outcomes than low- 
volume centers for pancreatectomy (24-26). Because most of the rural patients $(61 \%)$ were treated at low-volume centers and low-volume was found to be an independent predictor of poor outcome in this study, lack of access to high-volume centers contribute to the rural/urban disparities.

Long travel distance limits access to care for rural patients with cancer, especially given that rural patients have limited financial means. In a large cohort of 35,000 patients, Lin et al. reported that patients with node positive colon cancer who had to travel $\geq 250$ miles for their oncology care were only one-third as likely to receive adjuvant chemotherapy (27). We found that the overwhelming majority of rural patients $(71 \%)$ in our study had to seek care at a facility located at a great circle distance of $\geq 50$ miles from their homes, compared to only $20 \%$ for urban patients $(\mathrm{P}<0.0001)$, great circle distance was not an independent predictor of outcome. This observation was also noted in a large population-based analysis from the British Columbia (20). The reasons why travel distance did not have an impact on outcome following pancreatectomy remain elusive. This may be related to the limitation of the travel distance variable; it is uncertain whether the reporting hospital is where the patient receives non-surgical cancer care such as chemotherapy. Perhaps those who had to travel far had their treatment at low-volume centers while those who had their treatment nearby had it at a high-volume center. Although the relationship between great circle distance and hospital volume is not known, it will be the focus of our subsequent study.

Investigators are seeking ways to achieve outcome parity for rural patients with cancer. In a recent retrospective analysis of 36,995 patients enrolled in SWOG (Southwest Oncology Group) treatment trials, Unger et al. reported that uniform treatment strategies for 17 diverse types of cancer from across 50 states eliminated cancer outcome disparity between rural and urban patients (28). Although this seminal study does shed some insights on the rural/ urban disparity question, it does have limitations. Patients who are enrolled in clinical trials such as those for SWOG generally have socioeconomic attributes that allowed them to receive the intended treatments and appropriate follow-up. The study did not account for barriers such as limited access to screening, higher prevalence of poor prognostic factors and comorbid condition, limited access to specialized care, educational barriers, and transportation challenges (29-31). In essence, the compared populations were homogeneous for the various aforementioned factors, and therefore, the results are difficult to apply to the overall general population (29).

Distortion from confounders may strengthen the effect of rurality on outcomes. For example, when we compared the hazard ratio between rural and urban regions after adjusting our crude model to include only patient's demographics (i.e., gender, race, age), we found that rurality independently predicted a $10 \%$ increase risk of death (Model 1). However, when this model included SDoH (i.e., income, education) as possible confounders, the independent nature of rurality disappears (Model 2). When we adjusted the crude model to include only patient's demographics and access to care factors (i.e., insurance, hospital type, hospital volume, great circle distance) (Model 3) or patient's demographics and clinical factors (i.e., stage, grade, treatment, and comorbidity) (Model 4), rurality persists as being an independent predictor of outcome.

However, when we included $\mathrm{SDoH}$ along with all other potential confounders (Model 5), we found that rural residency no longer is an independent predictor of survival. Thus, our modeling suggests that the observed association between rurality and poor pancreatic cancer outcome in univariate analysis does not represent a true causal relationship, but is rather the result of the association of adverse SDoH. In other words, rurality is not the cause of the poor outcome observed for, but rather due to adverse $\mathrm{SDoH}$ and other related factors.

Our data demonstrated that poor outcome following a pancreatectomy for rural patients was not due to some intrinsic property of geography. In other words, when potential confounders are controlled, outcomes were not significantly different between rural and urban patients. However, disparities in OS do exist and these were mainly due to adverse SDoH that led to poor outcomes. These findings have important implications. By addressing these adverse $\mathrm{SDoH}$, it is possible to mitigate the rural/urban gap.

Our study does have several limitations. Patients may have received cancer treatment in more than one facility and therefore it is difficult to delineate the true impact of facility type. Another limitation is that the NCDB data are hospital-based, not population-based. Patients who received cancer care at hospitals accredited by $\mathrm{ACoS}$ may be different from those receiving care at non-ACoS facilities. The quality of cancer care may also be different between $\mathrm{ACoS}$ and non-ACoS facilities. Because ACoS hospitals provide cancer care to more than $70 \%$ of cancer patients in the United States, the findings using NCDB data are still meaningful. Referral patterns and how patients select their treatment center are not captured in the database. Another 
limitation is that the study does not account for possible migration of surgeons moving from one facility type to another. Factors such as tumor biology, chemotherapy dosage, compliance with chemotherapy and radiation therapy, and clinical trial participation were not captured in our study, which further limit our results. There are $1,928(7.6 \%)$ patients lost to follow-up (survival follow up after completion of surgery, chemo, RT, and so on). It is conceivable that patients with adverse SDoH may have contributed to the missing numbers lost for follow up. Finally, our analysis lacks cause-specific survival information since the cause of death was not captured in NCDB.

\section{Conclusions}

We report a disparity in outcome between rural and urban patients who underwent a pancreatectomy for resectable pancreatic adenocarcinoma. Such disparity, however, was associated with the adverse SDoH. Compared to urban patients, rural patients tend to be poorer, less likely to attain higher level of education, more underinsured, more likely to be treated at a community cancer program, and more likely to travel a long distance for their care. These identifiable adverse SDoH can be of value for policymakers, health care providers, and stakeholders as they address them to reach parity in outcomes for rural patients.

\section{Acknowledgments}

This paper was presented at the Annual Meeting of the 2020 Americas-Hepato-Pancreato-Biliary Association, March 5-8, 2020, Loews Miami Beach Hotel, Miami Beach, FL. The data used in the study are derived from a deidentified NCDB file. The American College of Surgeons and the Commission on Cancer have not verified and are not responsible for the analytic or statistical methodology employed, or the conclusions drawn from these data by the investigator. The authors are indebted to the learner's thoughtful discussion from the Thomas Jefferson College of Population Health.

Funding: The Edward \& Freda Green Endowed Professorship in Surgical Oncology supported this work.

\section{Footnote}

Reporting Checklist: The authors have completed the STROBE reporting checklist. Available at https://dx.doi. org/10.21037/jgo-20-583
Peer Review File: Available at https://dx.doi.org/10.21037/ jgo-20-583

Conflicts of Interest: All authors have completed the ICMJE uniform disclosure form (available at https://dx.doi. org/10.21037/jgo-20-583). The authors have no conflicts of interest to declare.

Ethical Statement: The authors are accountable for all aspects of the work in ensuring that questions related to the accuracy or integrity of any part of the work are appropriately investigate and resolved. The study was conducted in accordance with the Declaration of Helsinki (as revised in 2013), and was exempted from Institutional Review Board (IRB) approval by the Louisiana State University Health Sciences Center-Shreveport.

Open Access Statement: This is an Open Access article distributed in accordance with the Creative Commons Attribution-NonCommercial-NoDerivs 4.0 International License (CC BY-NC-ND 4.0), which permits the noncommercial replication and distribution of the article with the strict proviso that no changes or edits are made and the original work is properly cited (including links to both the formal publication through the relevant DOI and the license). See: https://creativecommons.org/licenses/by-nc-nd/4.0/.

\section{References}

1. Siegel RL, Miller KD, Jemal A. Cancer statistics, 2016. CA Cancer J Clin 2016;66:7-30.

2. Siegel RL, Miller KD, Jemal A. Cancer statistics, 2019. CA Cancer J Clin 2019;69:7-34.

3. Rahib L, Smith BD, Aizenberg R, et al. Projecting cancer incidence and deaths to 2030: the unexpected burden of thyroid, liver, and pancreas cancers in the United States. Cancer Res 2014;74:2913-21.

4. Siegel RL, Miller KD, Jemal A. Cancer statistics, 2020. CA Cancer J Clin 2020;70:7-30.

5. Henley SJ, Anderson RN, Thomas CC, et al. Invasive Cancer Incidence, 2004-2013, and Deaths, 2006-2015, in Nonmetropolitan and Metropolitan Counties - United States. MMWR Surveill Summ 2017;66:1-13.

6. Bolin JN, Bellamy GR, Ferdinand AO, et al. Rural Healthy People 2020: New Decade, Same Challenges. J Rural Health 2015;31:326-33.

7. Scarton L, Yoon S, Oh S, et al. Pancreatic Cancer Related Health Disparities: A Commentary. Cancers (Basel) 
2018;10:235.

8. Alcaraz KI, Wiedt TL, Daniels EC, et al. Understanding and addressing social determinants to advance cancer health equity in the United States: A blueprint for practice, research, and policy. CA Cancer J Clin 2020;70:31-46.

9. World Health Organization. Social determinants of health. Available online: https://www.who.int/social_ determinants/sdh_definition/en/. Accessed Nov 6, 2019.

10. Woolf SH. Progress In Achieving Health Equity Requires Attention To Root Causes. Health Aff (Millwood) 2017;36:984-91.

11. Kaplan RM, Milstein A. Contributions of Health Care to Longevity: A Review of 4 Estimation Methods. Ann Fam Med 2019;17:267-72.

12. Vick AD, Hery DN, Markowiak SF, et al. Closing the Disparity in Pancreatic Cancer Outcomes: A Closer Look at Nonmodifiable Factors and Their Potential Use in Treatment. Pancreas 2019;48:242-9.

13. Ashktorab H, Kupfer SS, Brim H, et al. Racial Disparity in Gastrointestinal Cancer Risk. Gastroenterology 2017;153:910-23.

14. Edge SB, Compton CC. The American Joint Committee on Cancer: the 7th edition of the AJCC cancer staging manual and the future of TNM. Ann Surg Oncol 2010;17:1471-4.

15. Categories of Accreditation. Available online: http://www. facs.org/cancer/coc/categories.html

16. Charlson ME, Pompei P, Ales KL, et al. A new method of classifying prognostic comorbidity in longitudinal studies: development and validation. J Chronic Dis 1987;40:373-83.

17. Deyo RA, Cherkin DC, Ciol MA. Adapting a clinical comorbidity index for use with ICD-9-CM administrative databases. J Clin Epidemiol 1992;45:613-9.

18. Papanicolas I, Woskie LR, Jha AK. Health Care Spending in the United States and Other High-Income Countries. JAMA 2018;319:1024-39.

19. Papanicolas I, Woskie LR, Jha AK. Comparing Spending on Medical Care in the United States and Other Countries-In Reply. JAMA 2018;320:840.

20. Canale TD, Cho H, Cheung WY. A populationbased analysis of urban-rural disparities in advanced

Cite this article as: Chu QD, Hsieh MC, Gibbs JF, Wu XC. Social determinants of health associated with poor outcome for rural patients following resected pancreatic cancer. J Gastrointest Oncol 2021;12(6):2567-2578. doi: 10.21037/jgo-20583 pancreatic cancer management and outcomes. Med Oncol 2018;35:116.

21. Kirkegård J, Ladekarl M, Fristrup CW, et al. Urban versus rural residency and pancreatic cancer survival: A Danish nationwide population-based cohort study. PLoS One 2018;13:e202486.

22. Murphy MM, Simons JP, Hill JS, et al. Pancreatic resection: a key component to reducing racial disparities in pancreatic adenocarcinoma. Cancer 2009;115:3979-90.

23. Singal V, Singal AK, Kuo YF. Racial disparities in treatment for pancreatic cancer and impact on survival: a population-based analysis. J Cancer Res Clin Oncol 2012;138:715-22.

24. Eppsteiner RW, Csikesz NG, McPhee JT, et al. Surgeon volume impacts hospital mortality for pancreatic resection. Ann Surg 2009;249:635-40.

25. Al-Refaie WB, Muluneh B, Zhong W, et al. Who receives their complex cancer surgery at low-volume hospitals? J Am Coll Surg 2012;214:81-7.

26. Chu QD, Zhou M, Peddi P, et al. Influence of facility type on survival outcomes after pan-createctomy for pancreatic adenocarcinoma. HPB (Oxford) 2017;19:1046-57.

27. Lin CC, Bruinooge SS, Kirkwood MK, et al. Association Between Geographic Access to Cancer Care, Insurance, and Receipt of Chemotherapy: Geographic Distribution of Oncologists and Travel Distance. J Clin Oncol 2015;33:3177-85.

28. Unger JM, Moseley A, Symington B, et al. Geographic Distribution and Survival Outcomes for Rural Patients With Cancer Treated in Clinical Trials. JAMA Netw Open 2018;1:e181235.

29. McCullough LE, Flowers CR. Identifying and Addressing Disparities in Survival Outcomes for Rural Patients With Cancer. JAMA Netw Open 2018;1:e181243.

30. Arcury TA, Preisser JS, Gesler WM, et al. Access to transportation and health care utilization in a rural region. J Rural Health 2005;21:31-8.

31. Glover S, Moore CG, Samuels ME, et al. Disparities in access to care among rural working-age adults. J Rural Health 2004;20:193-205. 